\title{
Verification of Software: The Textbook and Real Problems
}

\author{
Jan-Reneé Carlson* \\ NASA-Langley Research Center, Hampton, Virginia, 23681, USA
}

\begin{abstract}
The process of verification, or determining the order of accuracy of computational codes, can be problematic when working with large, legacy computational methods that have been used extensively in industry or government. Verification does not ensure that the computer program is producing a physically correct solution, it ensures merely that the observed order of accuracy of solutions are the same as the theoretical order of accuracy. The Method of Manufactured Solutions (MMS) is one of several ways for determining the order of accuracy. MMS is used to verify a series of computer codes progressing in sophistication from "textbook" to "real life" applications. The degree of numerical precision in the computations considerably influenced the range of mesh density to achieve the theoretical order of accuracy even for 1-D problems. The choice of manufactured solutions and mesh form shifted the observed order in specific areas but not in general. Solution residual (iterative) convergence was not always achieved for 2-D Euler manufactured solutions. $\mathbf{L}_{2, \text { norm }}$ convergence differed variable to variable therefore an observed order of accuracy could not be determined conclusively in all cases, the cause of which is currently under investigation.
\end{abstract}

\section{Nomenclature}

$\mathcal{S} \quad$ Source term

$C \quad$ Coefficient in manufactured solution

E Energy

$L, L_{x}, L_{y}$ Reference length

$N \quad$ Number of mesh nodes between computational boundaries

$p \quad$ Pressure

Re $1 / \nu$

$t \quad$ Time

$u, v \quad$ Cartesian velocity components

$x, y \quad$ Cartesian coordinates in physical space

$\mathrm{L}_{2, \text { norm }}$ vector norm

T.E. $1 \frac{1}{3}\left(\Delta x_{+}-\Delta x_{-}\right) \phi_{x x x}$, First term of truncation error, Eq. 12

T.E.2 $\frac{1}{12}\left(\frac{\Delta x_{+}^{3}+\Delta x_{-}^{3}}{\Delta x_{+}+\Delta x_{-}}\right) \phi_{x x x x}$, Second term of truncation error, Eq. 12

Subscripts

$0 \quad$ Initial mesh spacing at $\mathrm{x}=0$

MS Manufactured solution

$i \quad$ Node index

Symbols

$\gamma \quad$ gas constant, 1.4

$\Delta x \quad$ Inter-node spacing

* Senior Scientist, Computational AeroSciences Branch, Research and Technology Directorate, NASA-LaRC, Senior Member.

Copyright (c) 2006 by the American Institute of Aeronautics and Astronautics, Inc. The U.S. Government has a royalty-free license to exercise all rights under the copyright claimed herein for Governmental purposes. All other rights are reserved by the copyright owner. 


$\begin{array}{ll}\Delta x_{+} & \left(x_{i+1}-x_{i}\right) \\ \Delta x_{-} & \left(x_{i}-x_{i-1}\right) \\ \Delta x_{N} & \text { Equal interval spacing, } 1 / N+1 \\ \nu & \text { Kinematic viscosity } \\ \Omega & \text { Computational domain } \\ \partial \Omega & \text { Boundary of computational domain } \\ \phi & \text { Arbitrary function in physical space } \\ \rho & \text { Density }\end{array}$

\section{Introduction}

$A \begin{aligned} & \text { BROAD definition of the numerical verification of a program written to solve partial differential equations } \\ & \text { (PDE's) is succinctly stated by Knupp \& Salari }{ }^{1}\end{aligned}$

"The process by which one demonstrates that a PDE code correctly solves its governing equations."

Consider the Laplace equation, $\nabla^{2} \phi=0$, within some boundary $\Omega$ with a set of conditions on the boundary, $\left.\phi\right|_{\Omega}=g$. For this discussion we consider solutions using finite difference techniques rather than any of the many analytic techniques that exist for this equation. Discretized equations are approximations of derivatives arrived at typically through Taylor series expansions of, in this example, the dependent variable $\phi$ at some distance $\Delta \mathbf{x}$ from $\mathbf{x}$. One discrete expression for the first derivative of $\phi$, with order property $\Delta x$, can be written as,

$$
\frac{\mathrm{d} \phi}{\mathrm{d} x} \approx \frac{\phi(x+\Delta x)-\phi(x)}{\Delta x}+O(\Delta x)
$$

For Laplace's equation, an expression for the second derivative could be written as

$$
\frac{\mathrm{d}^{2} \phi}{\mathrm{d} x^{2}} \approx \frac{\phi(x+\Delta x)-2 \phi(x)+\phi(x-\Delta x)}{\Delta x^{2}}+O\left(\Delta x^{2}\right)
$$

That is, as $\Delta x$ is decreased, the discrete expression $\left(\phi(x+\Delta x)-2 \phi(x)+\phi(x-\Delta x) / \Delta x^{2}\right.$ should approach the exact expression $\mathrm{d}^{2} \phi / \mathrm{d} x^{2}$ at the rate of $\Delta x^{2}$. It is important therefore to verify that a program, written for example using Eq. 2 in the discretization of the second derivative, exhibit the correct second order property behavior.

The method of manufactured solutions is one of several ways to verify the order properties of numerical methods. Discussions of verification methods can be found in various books and reports, for example, by Roache $^{2}$, Salari \& Knupp ${ }^{3}$, Oberkampf \& Trucano ${ }^{4}$ and Roy et al. ${ }^{5}$

Briefly, consider the general form,

$$
\mathcal{D} \phi-\mathcal{S}=0
$$

where $\mathcal{D}$ and $\mathcal{S}$ are, respectively, a differential operator on $\phi$ and some source term. When using the method of manufactured solutions for verification, another source term, $\mathcal{S}_{\mathrm{MS}}$, is added to Eq. 3 as a forcing function

$$
\mathcal{D} \phi-\mathcal{S}=\mathcal{S}_{\mathrm{MS}}, \quad \text { where } \quad \mathcal{S}_{\mathrm{MS}}=[\mathcal{D} \phi-\mathcal{S}]_{\phi=\phi_{\mathrm{MS}}}
$$

The function $\mathcal{S}_{\mathrm{MS}}$ is a function consisting of the same differential operator as the modeled equation applied to the predetermined solution function, $\phi_{\mathrm{MS}}$. The difference between the iteratively converged discrete solution of Eq. 4 and the predetermined solution function is the discretization error and will be a function of the mesh spacing and the truncation error of the discretization scheme. The sum of the differences between the discrete solution and the predetermined solution over the solution space results in an integrated value for the discretization error $\mathrm{L}_{2, \text { norm }}{ }^{\mathrm{a}}$

$$
\mathrm{L}_{2, \mathrm{norm}}=\sqrt{\frac{\sum_{n=1}^{N}\left(\phi_{\text {solution }}-\phi_{\mathrm{MS}}\right)^{2}}{N}}
$$

This process is repeated for 3 or more mesh densities. The observed order property of the solution method is obtained from the change of the discretization error with mesh size.

${ }^{a}$ http://mathworld.wolfram.com/L2-Norm.html 


\section{1-D Poisson Equation}

The ubiquitous Poisson equation subject to Dirichlet boundary conditions, Eq. 6, serves as the initial platform for demonstrating text book verification, both successes and pitfalls, using manufactured solutions. The function $\mathbf{f}$ satisfies Eq. 6 throughout the interior of $\Omega$ subject to the physical source term $v$. The Dirichlet condition $\mathbf{f}=\mathbf{g}$ is set along the boundary $\partial \Omega$.

$$
\nabla^{2} \mathbf{f}-v=\mathcal{S}_{\mathrm{MS}}, \text { in } \Omega, \quad \mathbf{f}=\mathbf{g}, \text { on } \partial \Omega \text {. }
$$

The manufactured solution source term is defined in Eq. 7, where $\mathbf{f}_{\mathrm{MS}}$ and $v_{\mathrm{MS}}$ are specified functions.

$$
\mathcal{S}_{\mathrm{MS}}=\nabla^{2} \mathbf{f}_{\mathrm{MS}}-v_{\mathrm{MS}}
$$

Now consider the domain $\Omega$ to be a single dimension partitioned in to $N$ segments with the boundaries at $x_{0}$ and $x_{N+1}$ such that $x_{0} \leq x_{i} \leq x_{N+1}, 0 \leq i \leq N+1$.

\section{II.A. Three point discretization in physical space}

If $f$ is a function of $\phi$ in physical space $x$, the difference of slopes at the half nodes $i \pm 1 / 2$ can be used to calculate the second derivative of $f$.

$$
\left.f_{x x}\right|_{i}=\frac{\left.\phi_{x}\right|_{i+1 / 2}-\left.\phi_{x}\right|_{i-1 / 2}}{x_{i+1 / 2}-x_{i-1 / 2}}=\frac{2}{\Delta x_{+}+\Delta x_{-}}\left(\frac{\phi_{i+1}-\phi_{i}}{\Delta x_{+}}-\frac{\phi_{i}-\phi_{i-1}}{\Delta x_{-}}\right)
$$

using the averaging expressions

$$
\left.\phi_{x}\right|_{i+1 / 2}=\frac{\phi_{i+1}-\phi_{i}}{\Delta x_{+}},\left.\quad \phi_{x}\right|_{i-1 / 2}=\frac{\phi_{i}-\phi_{i}}{\Delta x_{-}}
$$

where $\phi_{i}=\phi\left(x_{i}\right), \Delta x_{+}=x_{i+1}-x_{i}$, and $\Delta x_{-}=x_{i}-x_{i-1}$. The Taylor series expansions of $\phi$ (Eq. 10) is substituted in to Eq. 8

$$
\begin{aligned}
\phi_{i \pm 1}=\phi_{i} \pm\left.\phi_{x}\right|_{i} \Delta x_{ \pm}+\left.\frac{1}{2} \phi_{x x}\right|_{i} \Delta x_{ \pm}^{2} & \pm\left.\frac{1}{6} \phi_{x x x}\right|_{i} \Delta x_{ \pm}^{3}+\left.\frac{1}{24} \phi_{x x x x}\right|_{i} \Delta x_{ \pm}^{4} \\
& \pm\left.\frac{1}{120} \phi_{x x x x x}\right|_{i} \Delta x_{ \pm}^{5}+\left.\frac{1}{720} \phi_{x x x x x x}\right|_{i} \Delta x_{ \pm}^{6} \pm O\left(\Delta x_{ \pm}^{7}\right)
\end{aligned}
$$

resulting in the expression for $f_{x x}$ (Eq. 11).

$$
\left.f_{x x}\right|_{i}=\phi_{x x}+\left.f_{x x}\right|_{\text {T.E. }}
$$

where truncation error terms of $\left.f_{x x}\right|_{\text {T.E. }}$ are

$$
\begin{array}{r}
\left.f_{x x}\right|_{\text {T.E. }}=\left.\frac{1}{3}\left(\Delta x_{+}-\Delta x_{-}\right) \phi_{x x x}\right|_{i}+\left.\frac{1}{12}\left(\frac{\Delta x_{+}^{3}+\Delta x_{-}^{3}}{\Delta x_{+}+\Delta x_{-}}\right) \phi_{x x x x}\right|_{i}+\left.\frac{1}{60}\left(\frac{\Delta x_{+}^{4}-\Delta x_{-}^{4}}{\Delta x_{+}+\Delta x_{-}}\right) \phi_{x x x x x}\right|_{i} \\
+\left.\frac{1}{360}\left(\frac{\Delta x_{+}^{5}+\Delta x_{-}^{5}}{\Delta x_{+}+\Delta x_{-}}\right) \phi_{x x x x x x}\right|_{i}+O\left(\Delta x_{ \pm}^{5}\right)
\end{array}
$$

If the mesh is uniformly distributed so that $\Delta x=\Delta x_{+}=\Delta x_{-}$, then Eqs. 11 and 12 reduce to the well known forms

$$
\left.f_{x x}\right|_{i}=\frac{\phi_{i+1}-2 \phi_{i}+\phi_{i-1}}{\Delta x^{2}}
$$

and

$$
\left.f_{x x}\right|_{\text {T.E. }}=\left.\frac{1}{12} \phi_{x x x x}\right|_{i} \Delta x^{2}+\left.\frac{1}{360} \phi_{x x x x x x}\right|_{i} \Delta x^{4}+O\left(\Delta x_{ \pm}^{6}\right)
$$

Equation 11 is formally only first order accurate in a non-uniformly spaced mesh since the leading term of Eq. 12 goes as $\Delta x$. In a uniformly spaced mesh, the leading term of Eq. 14 varies as $\Delta x^{2}$ so that the 
difference equation Eq. 13 is formally second order accurate. Though, as noted in Hoffman ${ }^{6}$, if the quantity $\left(\Delta x_{+}-\Delta x_{-}\right)$has the characteristic of decreasing by a factor of 4 for a halving of the mesh spacing, then Eq. 11 is second order accurate as well since the leading term in Eq. 12 would then decrease as $O\left(\Delta x^{2}\right)$.

Also discussed in Hoffman, ${ }^{6}$ but without detailed derivations, were expressions for the central difference expression for the second derivative in transformed space. The derivation is similar to the one in physical space just discussed and is detailed in Appendix A,

\section{II.B. Forms of Manufactured Solutions}

Several guidelines have been published concerning the form of the manufactured solutions to be used in verification of computational methods, see Roache ${ }^{2}$ or Knupp \& Salari. ${ }^{1}$ As far as the assessment of a methods order of accuracy is concerned, the most important feature for any manufactured solution is the truncation error should be non-zero.

\section{II.B.1. Exponential and Trigonometric Functions}

Exponential and trigonometric functions are often used in manufactured solutions. These functions are smooth, continuous with an infinite number of smooth and continuous higher order derivatives.

A simple example would be single exponential function

$$
f_{\mathrm{MS}}=e^{-x} \rightarrow \frac{d^{m} f_{\mathrm{MS}}}{d x^{m}}=(-1)^{m} e^{-x}
$$

as do trigonometric functions such as sin or cos, and hyperbolic functions such as sinh and tanh. For example ,

$$
f_{\mathrm{MS}}=C \sin \left(\frac{2 \pi x}{L}\right) \rightarrow \frac{d^{m} f_{\mathrm{MS}}}{d x^{m}}= \begin{cases}C(-1)^{\frac{m-1}{2}}\left(\frac{2 \pi}{L}\right)^{m} \cos \left(\frac{2 \pi x}{L}\right) & : \text { m odd } \\ C(-1)^{\frac{m}{2}}\left(\frac{2 \pi}{L}\right)^{m} \sin \left(\frac{2 \pi x}{L}\right) & : \text { m even }\end{cases}
$$

Using a sin function as the manufactured solution, Eq. 16, the MS source term is

$$
\mathcal{S}_{\mathrm{MS}}=-C\left(\frac{2 \pi}{L}\right)^{2} \sin \left(\frac{2 \pi x}{L}\right)-v_{\mathrm{MS}}
$$

\section{II.B.2. Polynomial Series}

It is also possible to use a polynomial in $\mathrm{x}$ to some power $n$ as a manufactured solution, for example Eq. 19.

$$
\begin{aligned}
& f_{\mathrm{MS}}=x^{n} \rightarrow\left[f_{\mathrm{MS}}\right]_{x x}=n(n-1) x^{n-2} \\
& v_{\mathrm{MS}}=v(x)
\end{aligned}
$$

The source term for the manufactured solution is then

$$
\left.\mathcal{S}_{\mathrm{MS}}\right|_{i}=n(n-1) x_{i}^{n-2}-\left.v_{\mathrm{MS}}\right|_{i}
$$

As mentioned previously, the manufactured solutions should be chosen such that the truncation error is non-zero for a finite $\Delta x$ in the modeled equations. A polynomial manufactured solution has to be of power $n=4$ or higher for a uniformly spaced mesh since the leading term of the truncation error is $1 / 12 \Delta x^{2} f_{x x x x}$. If $n<4$ the manufactured solution will be an exact solution of the discretized equations and not display any order property with mesh refinement. There is a potential benefit from this result and is discussed in a subsequent section.

\section{II.C. 1-D Verification Case Studies}

Changes ins $\mathrm{L}_{2, \text { norm }}$ of the observed order and truncation error with the form of manufactured solution, mesh distribution, stencil size and numerical precision using the 1-D Poisson equation is discussed in the following sections. The parameter variations are listed in Table 1; though not all the cases will be specifically discussed. 
Table 1. List of Case Studies

\begin{tabular}{cccl}
\hline Case & \multicolumn{1}{c}{$f_{\mathrm{MS}}$} & Stencil & Mesh Form \\
\hline 1 & $\sin (x)$ & 3 pt. & Uniform \\
2 & $\sin (x)$ & 3 pt. & Geometric series \\
3 & $x^{6}$ & 3 pt. & Uniform \\
4 & $x^{3}$ & 3 pt. & Uniform \\
5 & $x^{3}$ & 3 pt. & Geometric series \\
6 & $x^{6}, v \neq v_{\mathrm{MS}}$ & 3 pt. & Uniform \\
7 & $x^{3}, v \neq v_{\mathrm{MS}}$ & 3 pt. & Uniform \\
8 & $x^{3}$ & 3 pt. & Elliptic DE \\
9 & $x^{6}$ & 3 pt. & Elliptic DE \\
10 & $\sin (x)$ & 5 pt. & Uniform \\
\hline
\end{tabular}

${ }^{*}$ Differential Equation

II.C.1. Study 1 - A Textbook Solution

Table 2. Study 1 Parameters

\begin{tabular}{ccccc}
\hline Case & Symbol & $f_{\mathrm{MS}}$ & $v_{\mathrm{MS}}$ & Mesh \\
\hline real $* 4$ & $\bigcirc$ & $\sin (2 \pi x)$ & $v$ & Uniform \\
real $* 8$ & $\square$ & $\sin (2 \pi x)$ & $v$ & Uniform \\
\hline
\end{tabular}

Consider a uniformly space computational mesh, $\Delta x$ with Dirichlet conditions applied at the boundary points, $\left.\phi\right|_{x=0}=\left.\phi\right|_{x=L}=$ 0 , and a manufactured solution of the form $\sin (2 \pi x / L)$. Single (32-bit) and double (64bit) precision calculations were performed using a direct solver on the second order accurate 3 point stencil equations in physical space shown in Eq. 21. The precision of the program variables were explicitly defined using the real $* 4$ or real $* 8$ FORTRAN statement calls for the single and double precision calculations respectively. The source code was compiled with Intel F90 FORTRAN and executed on the $1.4 \mathrm{GHz}$ Opteron CPU.

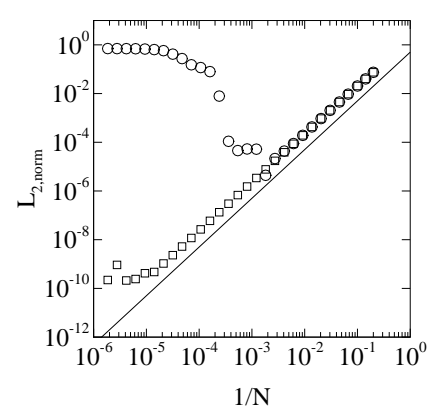

(a) $\mathrm{L}_{2, \text { norm }}$ vs. $1 / N$

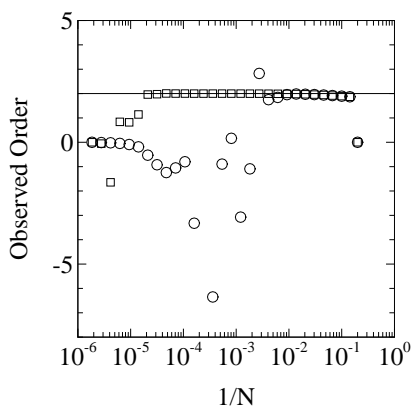

(b) Observed order vs. $1 / N$

Figure 1. 1-D Poisson equation, 3-point stencil, $2^{\text {nd }}$ order scheme, $\bigcirc, 32$-bit precision ; $\square, 64$-bit precision

$$
\begin{aligned}
& \phi_{i+1}-2 \phi_{i}+\phi_{i-1}= \\
& \quad-4 \pi^{2} \sin \left(2 \pi x_{i}\right) \Delta x^{2},\left.\quad v_{\mathrm{MS}}\right|_{i}=v_{i}
\end{aligned}
$$

The variation of the discretization error, $\mathrm{L}_{2}$,norm, with mesh spacing and the observed order of accuracy is shown in figure 1. The number of computational nodes ranged from 5 to 540,217 corresponding to a node-to-node spacing from approximately $1.7 \times 10^{-1}$ to $1.9 \times 10^{-6}$ based on a unit interval. The range also corresponds to a mesh ratio of $\mathrm{r}=1.5$ for 30 mesh levels starting at $\mathrm{N}=5$.

The design numerical order using Single precision arithmetic was limited in the range of mesh spacing due to accumulative round-off error. The use of double-precision arithmetic allowed for another 2.5 orders of magnitude reduction in mesh spacing and still retain textbook order of accuracy, figure 1(b). 
II.C.2. Study 2 - Difference with geometric series mesh expansion, Case 1 vs. Case 2

Table 3. Study 2 - Case 1 vs. Case 2

\begin{tabular}{cccclcc}
\hline Case & Symbol & $f_{\text {MS }}$ & $v_{\text {MS }}$ & Mesh & T.E.1 & T.E.2 \\
\hline 1 & $\circ$ & $\sin (2 \pi x)$ & $v$ & Uniform & 0 & $\frac{1}{12}(2 \pi)^{4} \Delta x^{2} \sin (2 \pi x)$ \\
2 & $\square, \diamond$ & $\sin (2 \pi x)$ & $v$ & $\begin{array}{l}\text { Geometric } \\
\text { series }\end{array}$ & $-\frac{1}{3}(2 \pi)^{3}\left(\Delta x_{+}-\Delta x_{-}\right) \cos (2 \pi x)$ & $\frac{1}{12}(2 \pi)^{4}\left(\frac{\Delta x_{+}^{3}+\Delta x_{-}^{3}}{\Delta x_{+}+\Delta x_{-}}\right) \sin (2 \pi x)$ \\
\hline
\end{tabular}

The mesh spacing for case 1 was $\Delta x=1 /(N+1)$ between nodes. For case 2 the spacing interval increased away from $x=0$ with the first interval $\Delta x_{0}=0.0001 /(N+1)$ for each of the meshes. A Newton iteration method was used to determine the correct expansion rate, r, to fit the $N$ nodes between the boundaries $0 \leq x \leq 1$ as defined in Eq. 22. For the cases studies here $L=1$.

$$
L=\Delta x_{0}\left(1+\sum_{i=1}^{N} r^{i}\right)
$$

The individual node positions were calculated using Eq. 23

$$
x_{i+1}=x_{i}+\Delta x_{0} r^{i}
$$

Table 10 in Appendix B details the total number of nodes, initial spacing and geometric expansion ratio for each mesh run. A direct solver was used to calculate solutions with Dirichlet boundary conditions for all 30 mesh density levels.

The variation of the discretization error, $\mathrm{L}_{2, \text { norm }}$, with mesh spacing and the observed order of accuracy for the two mesh distribution are compared in figures 2(a) and 2(b). Figure 2(c) is a plot of the $\mathrm{L}_{2, T . E \text {. of the non-zero trunca- }}$

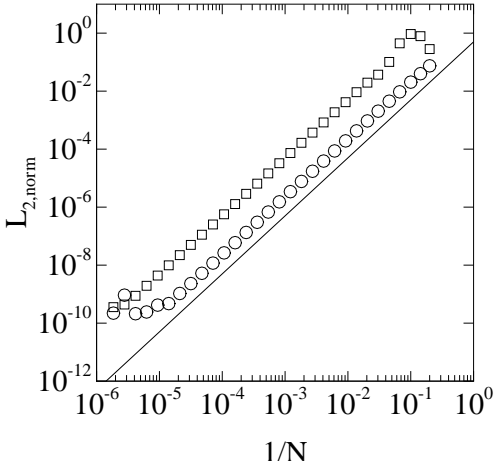

(a) $\mathrm{L}_{2, \text { norm }}$ vs. $1 / \mathrm{N}, \bigcirc$, Uniform mesh ; $\square$, Geometric series ; $-\mathrm{O}\left(\Delta x^{2}\right)$.

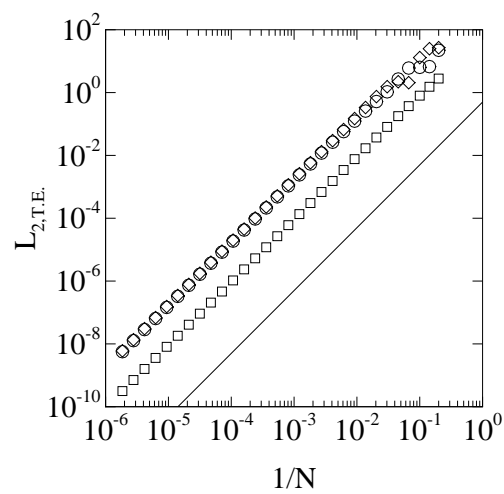

(c) $\mathrm{L}_{2, T . E}$ vs. $1 / \mathrm{N}, \bigcirc$, Case 1 - T.E.2 ; (d) $f_{x x}$, T.E. 1 vs. $\mathrm{x}, \mathrm{N}=163, ;$ $\square$, Case 2 - T.E.1 $; \diamond$, Case 2 - T.E.2 $; \mathrm{f}_{x x}$ discrete solution $;----$, T.E.1 $;-\cdot-\cdot$, $\longrightarrow, \mathrm{O}\left(\Delta x^{2}\right)$

Figure 2. 1-D Poisson equation, sinusoidal MS, $f_{\mathrm{MS}}=\sin (x)$, uniform vs. non-uniform mesh . tion error terms for the 2 cases and for the $N=163$ geometric series, the first two terms of truncation error mesh are shown in figure 2(d).

The uniform and geometric series mesh for $\approx 100<N<\approx 10,000$, both displayed with second order accurate behavior using a sinusoidal manufactured solution, comparing the $\bigcirc$ and $\square$ symbols in figures 2(a) and 2(b). The asymptotic range was roughly the same between the two mesh types-the uniformly spaced mesh approached order 2 in a more continuous fashion than the non-uniform meshes and departed from the design order due to numerical round off errors at a lower value of $\mathrm{N}$. The different trends of the $\mathrm{L}_{2}$ with increasing $\mathrm{N}$ is due to significantly higher truncation error of the non-uniform grid for meshes with less than 70 nodes. For $\mathrm{N}=73$ the geometric expansion factor was approximately 1.2, see Table 10.

The $\mathrm{L}_{2, \text { norm }}$ of T.E.2 were almost identical between the two cases, compare $\bigcirc$ and $\diamond$ symbols in figure 2(c). The first and second truncation error terms for case 2 are similar in magnitude, but $\pi$ radians out of phase, 
compare - - - with - - - in figure 2(d). The two truncation terms predominate in different regions of the solution space but overall the observed order is unaffected because both terms behave as $\Delta x^{2}$ in this case. , compare $\square$ and $\diamond$ symbols in figure $2(\mathrm{c})$.

II.C.3. Study 3 - Polynomial manufactured solutions on a uniform mesh, Case 3 vs. Case 4

Table 4. Study 3 - Case 3 vs. Case 4

\begin{tabular}{ccccccc}
\hline Case & Symbol & $f_{\text {MS }}$ & $v_{\text {MS }}$ & Mesh & T.E.1 & T.E. 2 \\
\hline 3 & $\bigcirc$ & $x^{6}$ & $v$ & Uniform & 0 & $30 \Delta x^{2} x^{2}$ \\
4 & $\square$ & $x^{3}$ & $v$ & Uniform & 0 & 0 \\
\hline
\end{tabular}

On the uniformly spaced mesh, the difference in the $\mathrm{L}_{2, \text { norm }}$ and the observed order between an order 6 polynomial and an order 3 polynomial manufactured solution is shown in figure 3. In the former case the MS source term will be an order 4 polynomial, while the latter choice the MS a linear function of $\mathrm{x}$.

The first term of the truncation error, T.E.1, is zero for both cases due to the uniformly spaced mesh and only case 3 has a non-zero second truncation term, T.E.2, since the fourth derivative of $x^{3}$ is zero. Therefore the correct order proper-

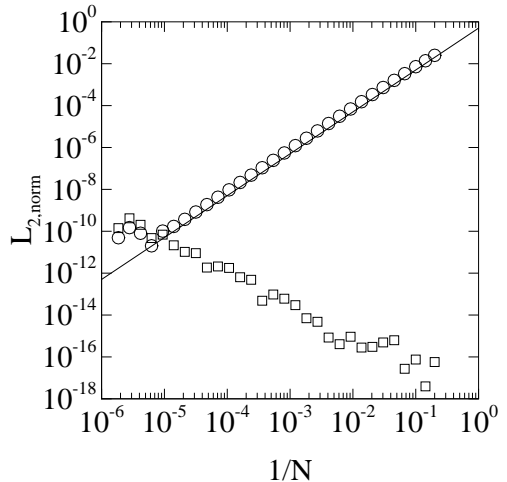

(a) $\mathrm{L}_{2, \text { norm }}$ vs. $1 / \mathrm{N}$.

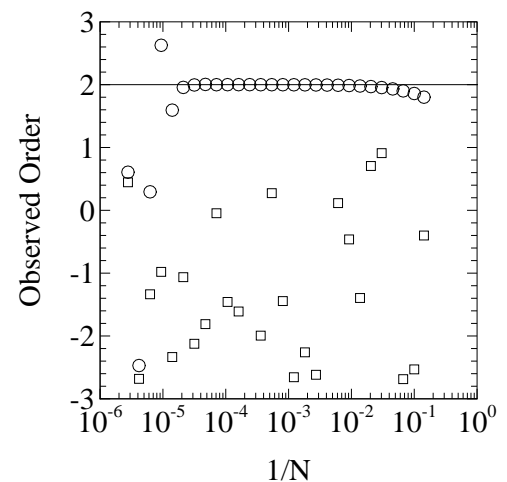

(b) Observed Order vs. $1 / \mathrm{N}$.

Figure 3. 1-D Poisson equation, uniform mesh, $\bigcirc, f_{\mathrm{MS}}=x^{6} ; \square, f_{\mathrm{MS}}=x^{3}$; , $\mathbf{O}\left(\Delta x^{2}\right)$ ties are seen only for the $f_{\mathrm{MS}}=x^{6}$ manufactured solution, see the $\bigcirc$ symbol in figures $3(\mathrm{a})$ and $3(\mathrm{~b})$.

The function $f_{\mathrm{MS}}=x^{3}$ as a manufactured solution is an exact solution of the discrete equations, hence the $\square$ symbol in figure 3(a) is representative of only accumulated round off error for $\mathrm{L}_{2}$,norm and as a result in figure 3(b), does not display an appropriate observed order.

II.C.4. Study 4-Polynomial manufactured solutions on a geometric series mesh, Case 4 vs. Case 5

Table 5. Study 4 - Case 4 vs. Case 5

\begin{tabular}{ccccccc}
\hline Case & Symbol & $f_{\text {MS }}$ & $v_{\text {MS }}$ & Mesh & T.E.1 & T.E.2 \\
\hline 4 & 0 & $x^{3}$ & $v$ & Uniform & 0 & 0 \\
5 & $\square$ & $x^{3}$ & $v$ & Geometric series & $2\left(\Delta x_{+}-\Delta x_{-}\right)$ & 0 \\
\hline
\end{tabular}

Case 5 used the same manufactured solution as did Case 4 but was solved on a non-uniformly spaced (geometric series ) mesh. An interesting result of this is that the first truncation error term, T.E.1, is nonzero and so an observed order should now be seen using a polynomial manufactured solution as low as order 3.

The $\mathrm{L}_{2}$,norm and observed order for cases 4 and 5 are compared in figures $4(\mathrm{a})$ and $4(\mathrm{~b})$. The $\mathrm{L}_{2}$ of the first truncation error term, T.E.1, for case 5 is plotted in figure 4(c).

Similar to what was discussed previously in section II.C.2, the quantity $\left(\Delta x_{+}-\Delta x_{-}\right)$is non-zero and has the appropriate behavior to give the term T.E.1 a second order convergence property with mesh refinement. Similar behavior seen in $\square$ symbols of figure 4 . 


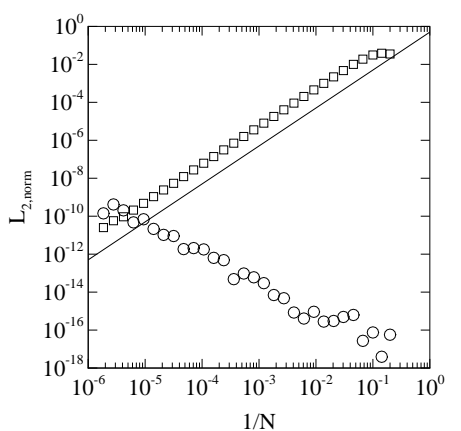

(a) $\mathrm{L}_{2, \text { norm }}$ vs. $1 / \mathrm{N}$.

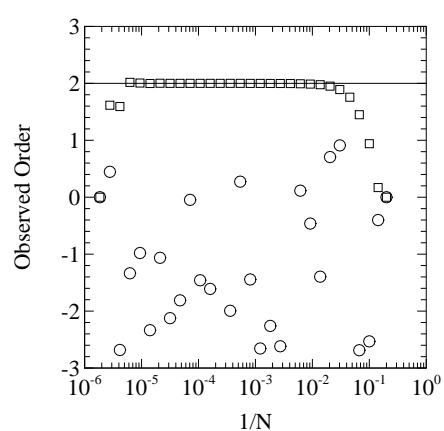

(b) Observed Order vs. $1 / \mathrm{N}$.

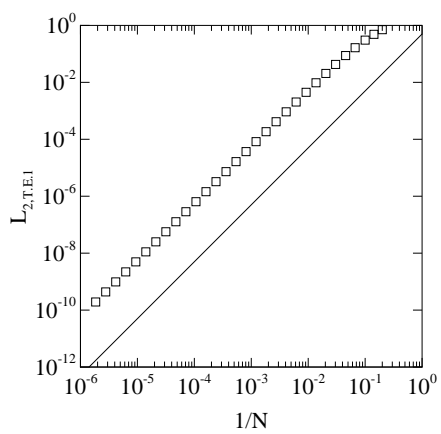

(c) $\mathrm{L}_{2, \text { T.E. } 1}$ vs. $1 / \mathrm{N}$.

Figure 4. 1-D Poisson equation, polynomial MS, $f_{\mathrm{MS}}=x^{3}$, $\bigcirc$, Uniform mesh ; $\square$, Geometric series mesh ; ,$- \mathbf{O}\left(\Delta x^{2}\right)$.

Table 6. Study 5 - Case 3 vs. Case 6

\begin{tabular}{ccccccc}
\hline Case & Symbol & $f_{\text {MS }}$ & $v_{\text {MS }}$ & Mesh & T.E.1 & T.E.2 \\
\hline 3 & $\circ$ & $x^{6}$ & $e^{-x}$ & Uniform & 0 & $30 \Delta x^{2} x^{2}$ \\
6 & $\square$ & $x^{6}$ & $e^{-1.00001 x}$ & Uniform & 0 & $30 \Delta x^{2} x^{2}$ \\
7 & $\diamond$ & $x^{3}$ & $e^{-1.00001 x}$ & Uniform & 0 & 0 \\
\hline
\end{tabular}

This section discusses introducing an error in the physical source term function $v$ of Eq. 6 . Previously, the manufactured solution $v_{\text {MS }}$ was identically $v$, so the source term was effectively "zeroed out" of the discretized equations. (a) $\mathrm{L}_{2, \text { norm }}$ vs. $1 / \mathrm{N}, f_{\mathrm{MS}}=x^{6}$, The case 6 and case 3 manufactured so-

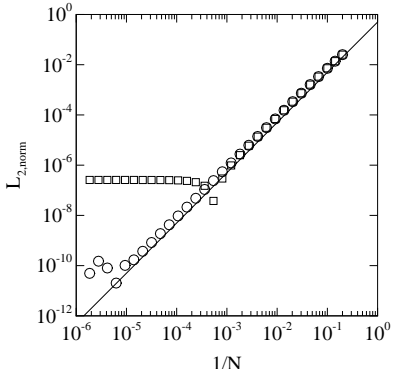

$\bigcirc, v_{\mathrm{MS}}=v, \square, v_{\mathrm{MS}} \neq v ;-$ $\mathrm{O}\left(\Delta x^{2}\right)$

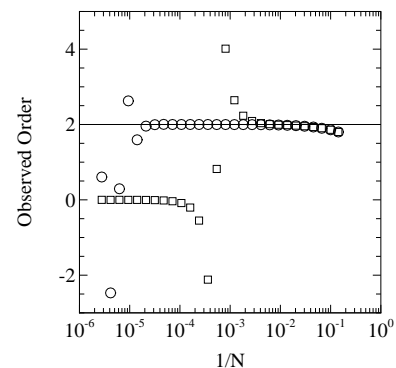

(b) Observed Order vs. $1 / \mathrm{N}$, (c) $\mathrm{L}_{2, \text { norm }}$ vs. $1 / \mathrm{N}, f_{\mathrm{MS}}=x^{3}$, $f_{\mathrm{MS}}=x^{6}, \bigcirc, v_{\mathrm{MS}}=v, \square, \diamond, v_{\mathrm{MS}} \neq v ;-, \mathrm{O}\left(\Delta x^{2}\right)$. $v_{\mathrm{MS}} \neq v ;-, \mathrm{O}\left(\Delta x^{2}\right)$.

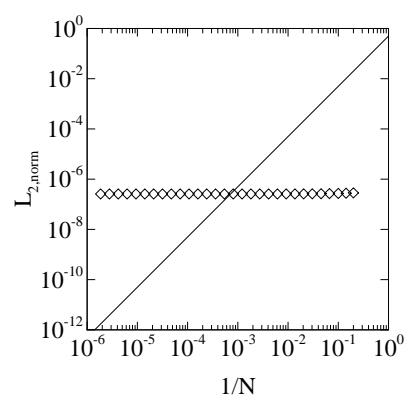

Figure 5. 1-D Poisson equation, uniform mesh, polynomial MS, $v_{\mathrm{MS}}=v$ vs. $v_{\mathrm{MS}} \neq v$. lution, $f_{\mathrm{MS}}$, and meshes in this sec-

tion were the same. The manufactured physical source term $v_{\text {MS }}$ was modified to mimic an error in $v$, shown in Table 6 . The $\mathrm{L}_{2, \text { norm }}$ and observed order for cases 3 and 6 are compared in figures $5(\mathrm{a})$ and $5(\mathrm{~b})$.

It is interesting to note that if the number of nodes in the order of accuracy study had been not exceeded $1000,{ }^{b}$ the departure from second order behavior for case 6 would not have been observed. For comparison, case 6 was repeated with an order 3 polynomial for the manufactured solution instead of the order 6 polynomial. It is known from the discussion in section II.C.3 that this combination of MS and mesh topology should produce $\mathrm{L}_{2 \text {,norm }}$ values close to floating point zero plus accumulated round off error. The significantly higher and constant $\mathrm{L}_{2, \text { norm }}$ shown in figure $5(\mathrm{c})$ is an indicator of the physical source term error.

Therefore the discontinuity in the $\mathrm{L}_{2 \text {,norm }}$ distribution around $1 / N \approx 10^{-3}$ for case $6, \square$ symbols in figure 5(a), is due to the physical model error. The same effect is observed in figure 5 (b) with the shift from the correct observed order 2 down to 0 for the $\square$.

\footnotetext{
${ }^{\mathrm{b}}$ The threshold of 1000 nodes is somewhat arbitrary in this case. The difference in the level of the $\mathrm{L}_{2 \text {, norm }}$ of the discretized equation compared with the $\mathrm{L}_{2}$,norm induced by the physical source term error would determine this.
} 
II.C.6. Study 6 - Polynomial manufactured solutions on a elliptic DE generated mesh, Case 3 vs. Case 9

Table 7. Study 6 - Case 3 vs. Case 9

\begin{tabular}{ccccccc}
\hline Case & Symbol & $f_{\text {MS }}$ & $v_{\text {MS }}$ & Mesh & T.E.1 & T.E.2 \\
\hline 3 & $\circ$ & $x^{6}$ & $v$ & Uniform & 0 & $30 \Delta x^{2} x^{2}$ \\
9 & $\square$ & $x^{6}$ & $v$ & Elliptic DE & $40\left(\Delta x_{+}-\Delta x_{-}\right) x^{3}$ & $30\left(\frac{\Delta x_{+}^{3}+\Delta x_{-}^{3}}{\Delta x_{+}+\Delta x_{-}}\right) x^{2}$ \\
\hline
\end{tabular}

The non-uniform mesh distribution results shown in sections II.C.2 and II.C.4 had the correct observed order due to the second order behavior of $\left(\Delta x_{+}-\Delta x_{-}\right)$with mesh refinement. The non-uniform mesh for Case 9 was generated using an elliptic differential equation, Eq. 24, instead of the ge-

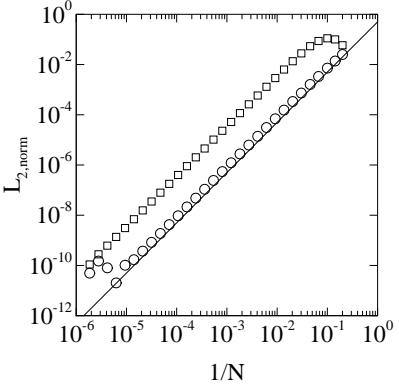

(a) $\mathrm{L}_{2, \text { norm }}$ vs. $1 / \mathrm{N}, \bigcirc$, Uniform mesh , $\square$, Elliptic DE mesh ; — $\mathrm{O}\left(\Delta x^{2}\right)$

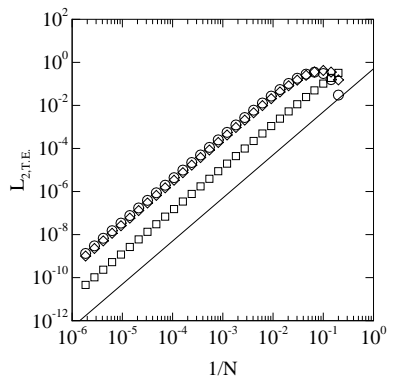

(b) Observed Order vs. $1 / \mathrm{N}, \bigcirc$, (c) $\mathrm{L}_{2, T}$.E vs. $1 / \mathrm{N}, \bigcirc$, Case 3 Uniform mesh , $\square$, Elliptic DE T.E.2 ; $\square$, Case 9 - T.E.1 ; $\diamond$, mesh ; - $\mathrm{O}\left(\Delta x^{2}\right)$.
Case 9 - T.E. $2 ;-, \mathrm{O}\left(\Delta x^{2}\right)$ ometric relation used in the previous sections. The coefficients of the function $P$ were chosen so as to cluster the nodes closer to $x=0$.

$$
\frac{d^{2} x}{d \xi^{2}}+P \frac{d x}{d \xi}=0
$$

The finite difference form used to generate the mesh and the clustering function was

$$
\left(1-\frac{P_{i}}{2}\right) x_{i-1}-2 x_{i}+\left(1+\frac{P_{i}}{2}\right) x_{i+1}=0, \quad P_{i}=-\frac{10}{N+1} e^{-i /(10(N+1))}
$$

Table 7 summarizes the parameters of this comparison study and Table 11 in Appendix B lists the initial mesh spacing with total node count. The $\mathrm{L}_{2, \text { norm }}$ and observed order for cases 3 and 9 are compared in figures 6(a) and 6(b). The $\mathrm{L}_{2}$ of the truncation error terms are shown in figure 6(c).

The coefficients used in the clustering function $\mathrm{P}$ were not optimized for any particular mesh. As can be seen in figures 6(a) and 6(b), $\square$ symbols, meshes with less than 50 nodes were not in the asymptotic range as the $\mathrm{L}_{2 \text {,norm }}$ distribution was nearly flat and the observed order was 1.9 or less. The clustering function was excessive for the more sparse meshes and could have been adjusted, though as far as this case study was concerned, wasn't really required as the design order of accuracy was still observed, just within a smaller range of mesh densities.

The relevant aspect of the truncation error terms for case 9, plotted in figure 6(c), is that both T.E.1 and T.E.2 have second order characteristics with increasing mesh density for $\mathrm{N}_{i} 50$. The elliptically generated mesh extended the range of asymptotic convergence to higher values of $N$ than with the uniformly spaced mesh. This result is likely academic as the particular node count and spacing would be unrealistic for most 3 -D meshes. 
II.C.7. Study 7 - A five-point stencil for the second derivative - Case 10

Table 8. Study 7 Parameters

\begin{tabular}{ccccc}
\hline Case & Symbol & $f_{\mathrm{MS}}$ & $v_{\mathrm{MS}}$ & Mesh \\
\hline real $* 4$ & 0 & $\sin (2 \pi x)$ & $v$ & Uniform \\
real $* 8$ & $\square$ & $\sin (2 \pi x)$ & $v$ & Uniform \\
\hline
\end{tabular}

The final case study for the 1-D Poisson problem investigated the order characteristics of a higher order accurate stencil. Similar to section II.C.1, single and double precision arithmetic computations are compared over a range of mesh densities. The $\mathrm{L}_{2, \text { norm }}$ and observed order are shown in figures $7(\mathrm{a})$ and $7(\mathrm{~b})$.

A penta-diagonal solver was used to resolve the discrete set of equations from applying a central 5 point finite difference stencil, Eq. 26, to a uniformly spaced physical grid.

$$
-\phi_{i-2}+16 \phi_{i-1}-30 \phi_{i}+16 \phi_{i+1}-\phi_{i+2}=\left.\mathrm{RHS}\right|_{i}
$$

Explicit boundary conditions were imposed at the end points modifying the first two and last two discretized equations were modified as shown in Eq. 27.

$$
\left(\begin{array}{l}
\left.R H S\right|_{1} \\
\left.R H S\right|_{2} \\
\vdots \\
\left.R H S\right|_{i} \\
\vdots \\
\left.R H S\right|_{N-1} \\
\left.R H S\right|_{N}
\end{array}\right)=\left(\begin{array}{l}
\left.12 \mathcal{S}\right|_{1} \Delta x_{N}^{2}+\left.\phi_{\mathrm{MS}}\right|_{-1}-\left.16 \phi_{\mathrm{MS}}\right|_{0} \\
\left.12 \mathcal{S}\right|_{2} \Delta x_{N}^{2}+\left.\phi_{\mathrm{MS}}\right|_{0} \\
\vdots \\
\left.12 \mathcal{S}\right|_{i} \Delta x_{N}^{2} \\
\vdots \\
\left.12 \mathcal{S}\right|_{N-1} \Delta x_{N}^{2}+\left.\phi_{\mathrm{MS}}\right|_{N+1} \\
\left.12 \mathcal{S}\right|_{N} \Delta x_{N}^{2}-\left.16 \phi_{\mathrm{MS}}\right|_{N+1}+\left.\phi_{\mathrm{MS}}\right|_{N+2}
\end{array}\right)
$$

It was surprising to observe the rather rapid departure from the design order of 4 with the use of single precision arithmetic, $\bigcirc$ symbol in figures $7(\mathrm{a})$ and $7(\mathrm{~b})$.

Double-precision arithmetic allowed for almost a 4 order reduction in mesh spacing and still retain the textbook order of accuracy, $\square$ symbol in figure $7(\mathrm{~b})$. So by comparison, the higher order stencil had a smaller range of applicability while retaining the design order of accuracy of the discretization scheme.

\section{2-D Burger's Equation}

Advancing in complexity, the second "textbook" problem was the advective-diffusive Burger's equation, where $u$ and $v$ are velocity components, and $R e$ is the inverse of the kinematic viscosity.

$$
\begin{aligned}
& u \frac{\partial u}{\partial x}+v \frac{\partial u}{\partial y}-\frac{1}{R e}\left(\frac{\partial^{2} u}{\partial x^{2}}+\frac{\partial^{2} u}{\partial y^{2}}\right)=\mathcal{S}_{u, \mathrm{MS}} \\
& u \frac{\partial v}{\partial x}+v \frac{\partial v}{\partial y}-\frac{1}{R e}\left(\frac{\partial^{2} v}{\partial x^{2}}+\frac{\partial^{2} v}{\partial y^{2}}\right)=\mathcal{S}_{v, \mathrm{MS}}
\end{aligned}
$$

The manufactured solutions and source terms had the form:

$$
u_{\mathrm{MS}}=u_{0}+\sin \left(\frac{2 \pi x}{L_{x}}\right)+\cos \left(\frac{2 \pi y}{L_{y}}\right), \quad v_{\mathrm{MS}}=v_{0}+\cos \left(\frac{2 \pi x}{L_{x}}\right)+\sin \left(\frac{2 \pi y}{L_{y}}\right)
$$




$$
\begin{aligned}
& \mathcal{S}_{u, \mathrm{MS}}=\left[u \frac{\partial u}{\partial x}+v \frac{\partial u}{\partial y}-\frac{1}{R e}\left(\frac{\partial^{2} u}{\partial x^{2}}+\frac{\partial^{2} u}{\partial y^{2}}\right)\right]_{u=u_{\mathrm{MS}}, v=v_{\mathrm{MS}}} \\
& \mathcal{S}_{v, \mathrm{MS}}=\left[u \frac{\partial v}{\partial x}+v \frac{\partial v}{\partial y}-\frac{1}{R e}\left(\frac{\partial^{2} v}{\partial x^{2}}+\frac{\partial^{2} v}{\partial y^{2}}\right)\right]_{u=u_{\mathrm{MS}}, v=v_{\mathrm{MS}}}
\end{aligned}
$$

where $u_{0}=v_{0}=0.5, L_{x}=L_{y}=\pi /(6 \lambda), \lambda=25$, and $R e=50$.

An 2-factor approximately factored scheme with $2^{\text {nd }}$ order, centraldifferencing was used to discretize the equations with pseudo-time stepping to obtain iterative convergence. The system of equations were solved using the cyclic Thomas algorithm along each direction. The computational domain was 2-dimensional with an even spaced mesh in the vertical and horizontal directions. Dirichlet boundary conditions were imposed on the four edges of the domain, $u=v=0$. This computer code approached the appropriate order property as the inverse mesh density, $1 / N=1 /\left(N_{x} \times N_{y}\right)$, approached 0.0001 denoted as the "asymptotic range" in figure 8 . The data below that of 2 is an indication of too coarse a mesh for the numerical scheme to retain the design order property.

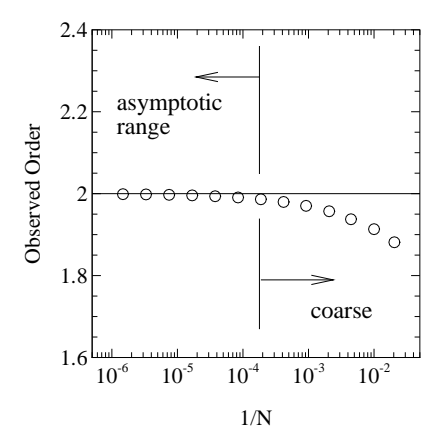

\section{2-D Euler Equations}

A legacy CFD code used in this section. The code was a multi-block,

Figure 8. 2-D Burger's Equation, Observed order vs. $1 / N$, double precision. $\bigcirc$, Code ; ,$- \mathrm{O}\left(\Delta x^{2}\right)$.

finite volume code that solves the three-dimensional compressible NavierStokes equations. The inviscid terms are upwind-biased spatial differenced. Solutions are developed using the flux-difference splitting procedure of $\mathrm{Roe}^{7}$ with MUSCL $^{8}$ (Monotone Upstream-center Scheme for Conservative Laws) first order, second or higher order differencing. The solution is advanced in time with a three-factor approximately factored scheme. For the initial verification of the code the 2-D Euler equations were solved, reducing the governing equations to eqs. 33 through 37.

$$
\begin{aligned}
& \frac{\partial \rho}{\partial t}+\frac{\partial \rho u}{\partial x}+\frac{\partial \rho v}{\partial y}=\mathcal{S}_{\text {continuity }, \mathrm{MS}} \\
& \frac{\partial \rho u}{\partial t}+\frac{\partial}{\partial x}\left[\rho u^{2}+p\right]+\frac{\partial}{\partial y}[\rho u v]=\mathcal{S}_{u, \mathrm{MS}} \\
& \frac{\partial \rho v}{\partial t}+\frac{\partial}{\partial x}[\rho v u]+\frac{\partial}{\partial y}\left[\rho v^{2}+p\right]=\mathcal{S}_{v, \mathrm{MS}} \\
& \frac{\partial}{\partial t}(\rho E)+\frac{\partial}{\partial x}[u(\rho E+p)]+\frac{\partial}{\partial y}[v(\rho E+p)]=\mathcal{S}_{\text {energy }, \mathrm{MS}} \\
& p=(\gamma-1)\left(\rho E-\frac{1}{2} \rho\left(u^{2}+v^{2}\right)\right)
\end{aligned}
$$

Forcing (or MS source) terms are generated from substituting the manufactured solutions in to the steady state forms of equations 33 through 36.

$$
\mathcal{S}_{\text {continuity }, \mathrm{MS}}=\frac{\partial}{\partial x_{j}}\left[\rho u_{j}\right], \quad \mathcal{S}_{u_{i}, \mathrm{MS}}=\frac{\partial}{\partial x_{j}}\left[\rho u_{i} u_{j}+p \delta_{i j}\right], \quad \mathcal{S}_{\text {energy }, \mathrm{MS}}=\frac{\partial}{\partial x_{j}}\left[u_{j}(\rho E+p)\right]
$$

The specific form of the manufactured solutions for the Navier-Stokes equations using density, velocity, 
and pressure are shown in Equations 39 through 42.

$$
\begin{aligned}
& \rho(x, y)=\rho_{0}+\rho_{x} \sin \left(\frac{a_{\rho x} \pi x}{L_{x}}\right)+\rho_{y} \cos \left(\frac{a_{\rho y} \pi y}{L_{y}}\right) \\
& u(x, y)=u_{0}+u_{x} \sin \left(\frac{a_{u x} \pi x}{L_{x}}\right)+u_{y} \cos \left(\frac{a_{u y} \pi y}{L_{y}}\right) \\
& v(x, y)=v_{0}+v_{x} \cos \left(\frac{a_{v x} \pi x}{L_{x}}\right)+v_{y} \sin \left(\frac{a_{v y} \pi y}{L_{y}}\right) \\
& p(x, y)=p_{0}+p_{x} \cos \left(\frac{a_{p x} \pi x}{L_{x}}\right)+p_{y} \sin \left(\frac{a_{p y} \pi y}{L_{y}}\right)
\end{aligned}
$$

$\mathcal{S}_{\text {continuity,MS }}, \mathcal{S}_{\text {momentum, MS }}$, and $\mathcal{S}_{\text {energy,MS }}$ are calculated by expanding eqs. 38 through 38 with eqs. 39 through 42 using the algebraic system Maple. ${ }^{\mathrm{C}}$

\section{IV.A. Solution Process}

Solutions were initialized with the MS functions, though this is not a preferred mode of operation as discussed in Knupp \& Salari. ${ }^{1}$ The solution discretization error is calculated each iteration for the primitive variables $\rho, \mathrm{u}, \mathrm{v}$ and $\mathrm{p}$ after the solution vector is updated.

The computational domain was two dimensional with an even spaced mesh in the vertical and horizontal directions. The domain was one unit in length in both dimensions with the reference lengths $L_{x}=L_{y}=1$. Periodic boundary conditions were imposed on the four edges of the domain. Euler flow solutions were run with no flux limiting.
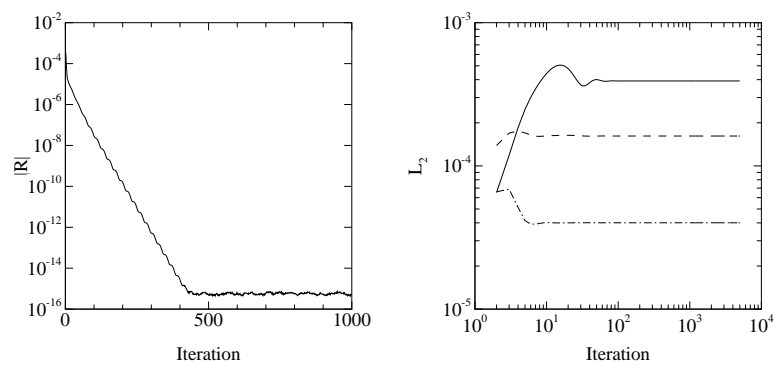

(a) $|R|$ vs. Iteration, $10 \times 10$ (b) $\mathrm{L}_{2}$ vs. Iteration, $10 \times 10$ mesh.

The solution residual and discretization error with iteration number for the $10 \times 10$ mesh for a $0.1 \%$ magnitude manufactured solution, for example, $\rho_{0} / \rho_{x}=\rho_{0} / \rho_{y}=0.001$ and all solutions were run with a $\mathrm{CFL}=8$. The results for the first-order discretization scheme are shown in figures 9(a) and 9(b). For these parameters iterative convergence to machine zero was obtained, figure 9 (a). Similarly the discretization error remained unchanged for several thousand iterations, though $\mathrm{L}_{2, p}$ converged in fewer iterations than $\mathrm{L}_{2, \rho}$. The appropriate first order behavior was observed for these parameters as shown in figure 9(c) and figure 9(d).

Manufactured solutions were repeated using a

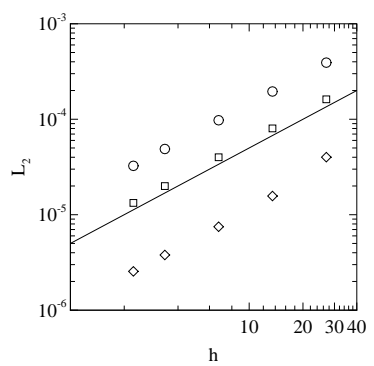

(c) $\mathrm{L}_{2, \rho}$ vs. $h$; Code $\bigcirc, \rho$; $\square$, (d) Order vs. $h$; Code $\bigcirc, \rho$; $u ; \diamond, p . ;-, O(\Delta x) . \quad \square, u ; \diamond, p$.

Figure 9. First-order scheme verification, 0.1\% magnitude MS, Euler flow, $\mathrm{CFL}=8$, no limiting. higher order option, $\kappa=1 / 3$, in the MUSCL scheme. Upon initial inspection of the results, figure $10(\mathrm{c})$, the code appeared to be verifying $3^{r d}$ order

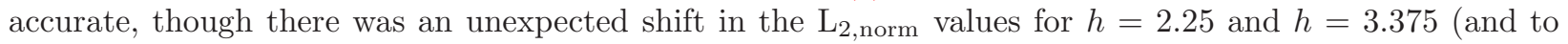
a lesser degree $h=6.75)$. The u-component of velocity and pressure displayed similar trends as density comparing the $\square$ and $\diamond$ with the $\bigcirc$ symbols in figure $10(\mathrm{c})$.

The discretization errors with solution iteration of density, u-velocity and pressure for each mesh densities are plotted in figure 11. It appears that the deviation of the $\mathrm{L}_{2, \text { norm }}$ for the density and velocity is due to the non-convergence of the discretization error at the higher mesh densities. The discretization error of the pressure was the only apparently converged quantity when only the discretization error is inspected.

\footnotetext{
${ }^{\mathrm{c}}$ Maple is a symbol computation system. Further information can be found at http://www.maplesoft.com/ .
} 

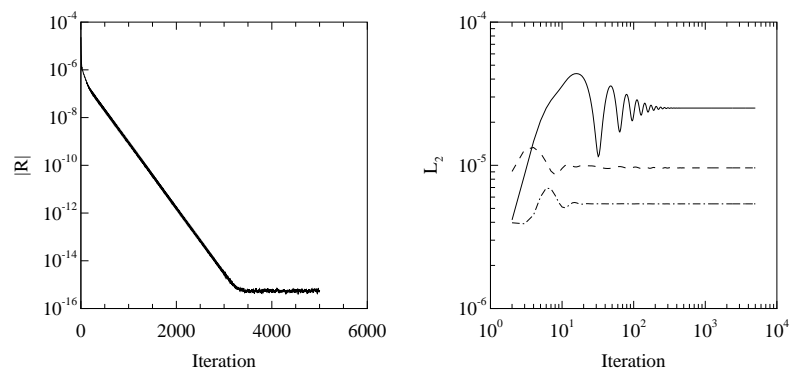

(a) $|R|$ vs. Iteration, $10 \times 10$ (b) $\mathrm{L}_{2}$ vs. Iteration, $10 \times 10$ mesh mesh ; -,$\rho ;---, u ;-\cdot-\cdot$,

$p$
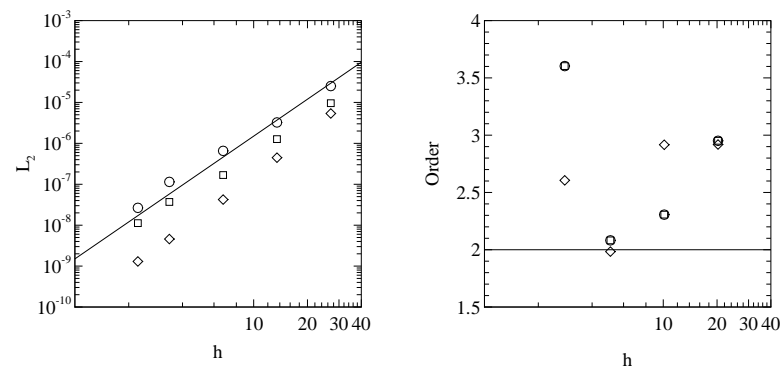

(c) $\mathrm{L}_{2, \rho}$ vs. $h$; Code $\bigcirc, \rho$; $\square$, (d) Order vs. $h$; Code $\bigcirc, \rho$; $u ; \diamond, p ;-, O\left(\Delta x^{3}\right) . \quad \square, u ; \diamond, p$.

Figure 10. Higher-order MUSCL scheme $(\kappa=1 / 3)$ verification, $\mathbf{0 . 1 \%}$ magnitude MS, Euler flow, $\mathrm{CFL}=8$, no limiting.

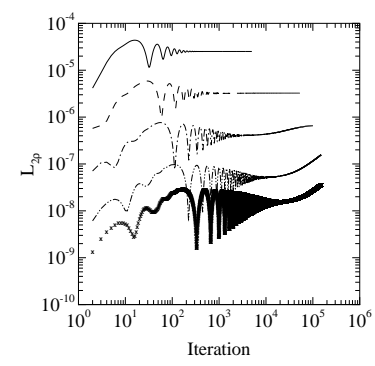

(a) $\mathrm{L}_{2, \rho}$ vs. Iteration

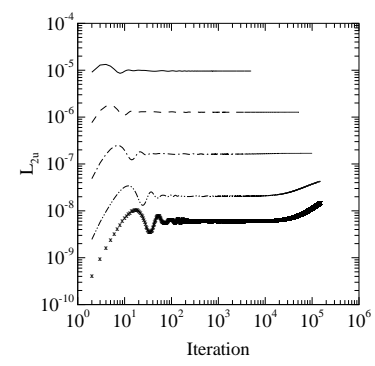

(b) $\mathrm{L}_{2, u}$ vs. Iteration

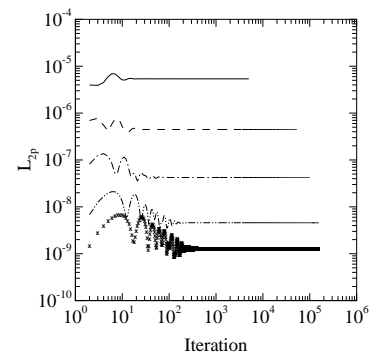

(c) $\mathrm{L}_{2, p}$ vs. Iteration

Figure 11. Convergence of discretization error with mesh density, $0.1 \%$ magnitude MS, Euler flow, CFL $=8$, $\kappa=1 / 3$, no limiting. ; -, $10 \times 10 ;-\cdot-, 20 \times 20 ;-\cdot-\cdot, 40 \times 40 ;-\cdots-\cdot, 80 \times 80 ; \mathbf{x}, 120 \times 120$

Only for the coarser mesh densities, (_- $10 \times 10$ and (---) $20 \times 20$, are iteratively converged in the solution residual plots, fig. 12. Potentially, after a sufficiently large number of iterations, the higher density meshes would be iteratively converged in the discretization error as well as the solution residual, but the wall-clock execution times would become unrealistic for routine testing. Table 9 lists the approximate run times for each of the mesh levels with MS. A per-iteration timing was roughly 20 milliseconds-per-point.

There are several (at this time unresolved) possibilities that could prevent the method from verifying. Problems could exist with, but not limited to, the implementation of the manufactured solutions in the code, the construction of the higher-order fluxes, the construction of the implicit terms or boundary conditions.

\section{Summary}

Verification is an important step in the evaluation of a numerical method and the concept is fairly straightforward. The implementation and analysis though are often problematic and require understanding the method of verification as well as the method being verified.

The method of manufactured solutions was applied to "textbook" and "real world" problems. Arithmetic precision was found to be a relevant factor for even a simple textbook problem. Additionally the design of 
Table 9. Timing for Manufactured Solutions Using 2-D Euler Method

\begin{tabular}{crrr}
\hline Mesh & $\begin{array}{r}\text { Iter. time } \\
(1 / \mathrm{sec})\end{array}$ & Iterations & $\begin{array}{c}\text { Total } \\
\text { time }\end{array}$ \\
\hline $10 \times 10$ & 0.002 & 5000 & $10 \mathrm{sec}$. \\
$20 \times 20$ & 0.008 & 50000 & $7 \mathrm{~min}$. \\
$40 \times 40$ & 0.032 & 150000 & $1.3 \mathrm{hr}$. \\
$80 \times 80$ & 0.128 & 150000 & $5.3 \mathrm{hr}$. \\
$120 \times 120$ & 0.288 & 150000 & $12 \mathrm{hr}$. \\
\hline
\end{tabular}

the discretization template influenced the order of accuracy analysis as did the mesh form and form of the manufactured solutions, though the departure from design order occurred in specific detail not in general trends.

With increasing size and complexity of the method to be verified, potentially the parameters that require analysis and/or inspection need to be increased as well. In the final example of the verification of the Euler equations in the Navier-Stokes solver, the non-convergence of the discretization error could have been over-looked if either only the behavior of the pressure was analyzed or the solutions stopped prematurely. Also, decisions must be made as to the time and resources to be devoted the verification of a code. The larger mesh densities required more resources and the run times quickly become unrealistic for efficient or routine reverification processes.

\section{A. Three point discretization in transformed space}

An expression for the truncation error of a first derivative expression of $f$ in the transformed coordinate $\xi$ was derived in Hoffman. ${ }^{6}$ Using that methodology the truncation error of the second derivative of $f$ in the transformed space is derived. Consider

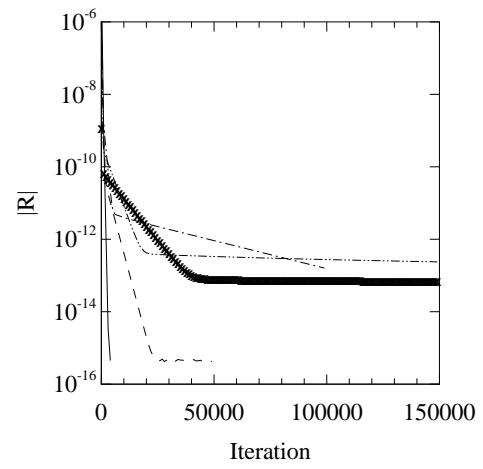

Figure 12. Variation of solution residual with mesh, MUSCL scheme $\kappa=1 / 3, \mathbf{0 . 1 \%}$ magnitude MS, Euler flow, $\mathrm{CFL}=8$, no limiting. ;, 10 x $10 ;---, 20 \times 20 ;-.-\cdot, 40 \times 40$ $;-\cdots-\cdot, 80 \times 80 ; \mathrm{x}, 120 \times 120$

$$
f=\phi(x)=\phi(g(\xi))=\psi(\xi)
$$

where the physical, $x$, and transformed, $\xi$, coordinates are functions of the form,

$$
\xi=h(x), \quad x=g(\xi)
$$

The second derivative $f_{x x}$ in the transformed coordinates is

$$
\begin{aligned}
f_{x x} & =(\psi(\xi))_{x x} \\
& =\xi_{x}\left(\xi_{x} \psi_{\xi}\right)_{\xi} \\
& =\xi_{x}^{2} \psi_{\xi \xi}+\xi_{x} \psi_{\xi} \xi_{x \xi}
\end{aligned}
$$

with the metrics for this $1-\mathrm{D}$ problem reducing to

$$
\left.x_{\xi}\right|_{i}=\frac{1}{2}\left(x_{i+1}-x_{i-1}\right),\left.\quad \xi_{x}\right|_{i}=\left.\frac{1}{x_{\xi}}\right|_{i}=\frac{2}{x_{i+1}-x_{i-1}}
$$

As defined previously $\Delta x_{+}=x_{i+1}-x_{i}$ and $\Delta x_{-}=x_{i}-x_{i-1}$, so that

$$
x_{i+1}-x_{i-1}=\left(\Delta x_{+}-\Delta x_{-}\right)
$$

resulting in the expression for the metric $\xi_{x}$.

$$
\left.\xi_{x}\right|_{i}=\frac{2}{\left(\Delta x_{+}-\Delta x_{-}\right)}
$$


A discretized equation for $f_{x x}$ can be derived from either Eq. A4 or Eq. A5. Expansion of Eq. A4 about the half nodes is written as

$$
\begin{aligned}
\left.\overline{\bar{f}}_{x x}\right|_{i} & =\left(\xi_{x}\left(\xi_{x} \psi_{\xi}\right)_{\xi}\right)_{i} \\
& =\left.\xi_{x}\right|_{i} \frac{1}{\Delta \xi}\left(\left.\left.\xi_{x}\right|_{i+1 / 2} \psi_{\xi}\right|_{i+1 / 2}-\left.\left.\xi_{x}\right|_{i-1 / 2} \psi_{\xi}\right|_{i-1 / 2}\right) \\
\Delta \xi & =\Delta \xi_{+}=\xi_{i+1}-\xi_{i}=\Delta \xi_{-}=\xi_{i}-\xi_{i-1}
\end{aligned}
$$

where the value of the metrics at the half nodes $x_{i+1 / 2}$ and $x_{i-1 / 2}$ are taken as the numerical average of the metrics at the neighboring nodes

$$
\left.\xi_{x}\right|_{i-1 / 2}=\frac{1}{2}\left(\left.\xi_{x}\right|_{i}+\left.\xi_{x}\right|_{i-1}\right),\left.\quad \xi_{x}\right|_{i+1 / 2}=\frac{1}{2}\left(\left.\xi_{x}\right|_{i+1}+\left.\xi_{x}\right|_{i}\right)
$$

and the derivatives $\left.\psi_{\xi}\right|_{i \pm 1 / 2}$ are

$$
\left.\psi_{\xi}\right|_{i+1 / 2}=\frac{\psi_{i+1}-\psi_{i}}{\Delta \xi},\left.\quad \psi_{\xi}\right|_{i-1 / 2}=\frac{\psi_{i}-\psi_{i-1}}{\Delta \xi}
$$

Cell-centered finite volume computational methods typically define the metrics of the mesh at nodes between cell centers eliminating the need for Eq. A12.

As an aside, if instead we start with equations A14, A15, and A16 in the transformed space and substitute in to Eq. A5,

$$
\begin{aligned}
\psi_{\xi \xi} & =\frac{\psi_{i+1}-2 \psi_{i}+\psi_{i-1}}{\Delta \xi^{2}} \\
\psi_{\xi} & =\frac{\psi_{i+1 / 2}-\psi_{i-1 / 2}}{\Delta \xi} \\
\xi_{x \xi} & =\frac{\left.\xi_{x}\right|_{i+1 / 2}-\left.\xi_{x}\right|_{i-1 / 2}}{\Delta \xi}
\end{aligned}
$$

we can write

$$
\begin{aligned}
\left.\overline{\bar{f}}_{x x}\right|_{i} & =\left(\xi_{x}^{2} \psi_{\xi \xi}+\xi_{x} \psi_{\xi} \xi_{x \xi}\right)_{i} \\
& =\left.\xi_{x}^{2}\right|_{i} \frac{\psi_{i+1}-2 \psi_{i}+\psi_{i-1}}{\Delta \xi^{2}}+\left.\left.\xi_{x}\right|_{i} \frac{\psi_{i+1 / 2}-\psi_{i-1 / 2}}{\Delta \xi} \xi_{x \xi}\right|_{i}
\end{aligned}
$$

Expanding $\xi_{x \xi}$ and assuming

$$
\psi_{i+1 / 2}=\frac{1}{2}\left(\psi_{i+1}+\psi_{i}\right), \quad \psi_{i-1 / 2}=\frac{1}{2}\left(\psi_{i}+\psi_{i-1}\right)
$$

the final form of the second derivative in the transformed space is equivalent to the previous expression written for $\overline{\bar{f}}_{x x}$ though the truncation error differs between the two methods.

The truncation error associated with Eq. A10 can be derived by expanding $\psi$ in the transformed space and substituting in to Eq. A5 using the discrete forms in Eqs. A14 and A15. Performing the same Taylor series expansion, here on $\psi(\xi)$,

$$
\begin{aligned}
& \psi_{i \pm 1}=\psi_{i} \pm\left.\psi_{\xi}\right|_{i} \Delta \xi+\left.\frac{1}{2} \psi_{\xi \xi}\right|_{i} \Delta \xi^{2} \pm\left.\frac{1}{6} \psi_{\xi \xi \xi}\right|_{i} \Delta \xi^{3}+\left.\frac{1}{24} \psi_{\xi \xi \xi \xi}\right|_{i} \Delta \xi^{4} \\
& \pm\left.\frac{1}{120} \psi_{\xi \xi \xi \xi \xi}\right|_{i} \Delta \xi^{5}+\left.\frac{1}{720} \psi_{\xi \xi \xi \xi \xi \xi}\right|_{i} \Delta \xi^{6} \pm O\left(\Delta \xi^{7}\right)
\end{aligned}
$$

Substituting Eq. A20 in to Eq. A18 averaging $\psi$ using the Eq. A19

$$
\begin{array}{r}
\left.\overline{\bar{f}}_{x x}\right|_{i}=\left(\xi_{x}^{2} \psi_{\xi \xi}+\xi_{x} \psi_{\xi} \xi_{x \xi}\right)_{i}+\left(\frac{1}{12} \xi_{x}^{2} \psi_{\xi \xi \xi \xi}+\frac{1}{6} \xi_{x} \psi_{\xi \xi \xi} \xi_{x \xi}\right)_{i} \Delta \xi^{2}+\left(\frac{1}{360} \xi_{x}^{2} \psi_{\xi \xi \xi \xi \xi \xi}+\frac{1}{120} \xi_{x} \psi_{\xi \xi \xi \xi \xi} \xi_{x \xi}\right)_{i} \Delta \xi^{4} \\
+\frac{1}{5040}\left(\xi_{x} \psi_{\xi \xi \xi \xi \xi \xi \xi} \xi_{x \xi}\right)_{i} \Delta \xi^{6}+O\left(\Delta \xi^{8}\right) \quad(\mathrm{A} 21)
\end{array}
$$


So that the expression

$$
\left.\overline{\bar{f}}_{x x}\right|_{i}=\frac{\left.\xi_{x}\right|_{i}}{\Delta \xi^{2}}\left[\left.\left(\psi_{i+1}-\psi_{i}\right) \xi_{x}\right|_{i+1 / 2}-\left.\left(\psi_{i}-\psi_{i-1}\right) \xi_{x}\right|_{i-1 / 2}\right]
$$

has the truncation error

$$
\begin{aligned}
\left.\overline{\bar{f}}_{x x}\right|_{\mathrm{TE}}=\left(\frac{1}{12} \xi_{x}^{2} \psi_{\xi \xi \xi \xi}+\frac{1}{6} \xi_{x} \psi_{\xi \xi \xi} \xi_{x \xi}\right)_{i} \Delta \xi^{2}+\left(\frac{1}{360} \xi_{x}^{2} \psi_{\xi \xi \xi \xi \xi \xi}+\frac{1}{120} \xi_{x} \psi_{\xi \xi \xi \xi \xi} \xi_{x \xi}\right)_{i} \Delta \xi^{4} \\
+\frac{1}{5040}\left(\xi_{x} \psi_{\xi \xi \xi \xi \xi \xi \xi \xi} \xi_{x \xi}\right)_{i} \Delta \xi^{6}+O\left(\Delta \xi^{8}\right)
\end{aligned}
$$

The alternative derivation expands the Taylor series about the half nodes $i \pm 1 / 2$,

$$
\psi_{i \pm 1 / 2}=\psi_{i} \pm\left.\psi_{\xi}\right|_{i}\left(\frac{\Delta \xi}{2}\right)+\left.\frac{1}{2} \psi_{\xi \xi}\right|_{i}\left(\frac{\Delta \xi}{2}\right)^{2} \pm\left.\frac{1}{6} \psi_{\xi \xi \xi}\right|_{i}\left(\frac{\Delta \xi}{2}\right)^{3}+\left.\frac{1}{24} \psi_{\xi \xi \xi \xi}\right|_{i}\left(\frac{\Delta \xi}{2}\right)^{4} \pm O\left(\Delta \xi^{5}\right)
$$

and substituting in to Eq. A18 (written only to $O\left(\Delta \xi^{4}\right)$ ).

$$
\left.\overline{\bar{f}}_{x x}\right|_{i}=\left(\xi_{x}^{2} \psi_{\xi \xi}+\xi_{x} \psi_{\xi} \xi_{x \xi}\right)_{i}+\left(\frac{1}{12} \xi_{x}^{2} \psi_{\xi \xi \xi \xi}+\frac{1}{24} \xi_{x} \psi_{\xi \xi \xi} \xi_{x \xi}\right)_{i} \Delta \xi^{2}+O\left(\Delta \xi^{4}\right)
$$

The difference between Eqs. A24 and A25 is the smaller numerical coefficient in the $\xi_{x} \psi_{\xi \xi \xi} \xi_{x \xi}$ term which slightly shifts the level of the truncation error but does not alter the order of the term.

The discrete equation for $\overline{\bar{f}}_{x x}$ in the transformed space is formally second order in $\Delta \xi$ for either method of forming $\psi_{x}$.

\section{B. 1-D Mesh Parameters}


Table 10. Geometric Series Mesh Parameters

\begin{tabular}{crrr|rrrr}
\hline Grid number & $N$ & $0.0001 \Delta x_{N}$ & $r$ & Grid number & $N$ & $0.0001 \Delta x_{N}$ & $r$ \\
\hline 1 & 5 & $1.667 \times 10^{-5}$ & 8.8139 & 16 & 1851 & $5.400 \times 10^{-8}$ & 1.0063 \\
2 & 7 & $1.250 \times 10^{-5}$ & 4.8543 & 17 & 2776 & $3.601 \times 10^{-8}$ & 1.0042 \\
3 & 10 & $9.091 \times 10^{-6}$ & 3.0691 & 18 & 4164 & $2.401 \times 10^{-8}$ & 1.0028 \\
4 & 15 & $6.250 \times 10^{-6}$ & 2.1311 & 19 & 6246 & $1.601 \times 10^{-8}$ & 1.0019 \\
5 & 22 & $4.348 \times 10^{-6}$ & 1.6823 & 20 & 9369 & $1.067 \times 10^{-8}$ & 1.0012 \\
6 & 33 & $2.941 \times 10^{-6}$ & 1.4175 & 21 & 14053 & $7.115 \times 10^{-9}$ & 1.0008 \\
7 & 49 & $2.000 \times 10^{-6}$ & 1.2661 & 22 & 21079 & $4.743 \times 10^{-9}$ & 1.0006 \\
8 & 73 & $1.351 \times 10^{-6}$ & 1.1722 & 23 & 31618 & $3.163 \times 10^{-9}$ & 1.0004 \\
9 & 109 & $9.091 \times 10^{-7}$ & 1.1125 & 24 & 47427 & $2.109 \times 10^{-9}$ & 1.0002 \\
10 & 163 & $6.098 \times 10^{-7}$ & 1.0740 & 25 & 71140 & $1.406 \times 10^{-9}$ & 1.0002 \\
11 & 244 & $4.082 \times 10^{-7}$ & 1.0489 & 26 & 106710 & $9.371 \times 10^{-10}$ & 1.0001 \\
12 & 366 & $2.725 \times 10^{-7}$ & 1.0324 & 27 & 160065 & $6.247 \times 10^{-10}$ & 1.0001 \\
13 & 549 & $1.818 \times 10^{-7}$ & 1.0215 & 28 & 240097 & $4.165 \times 10^{-10}$ & $<1.0001$ \\
14 & 823 & $1.214 \times 10^{-7}$ & 1.0143 & 29 & 360145 & $2.777 \times 10^{-10}$ & $<1.0001$ \\
15 & 1234 & $8.097 \times 10^{-8}$ & 1.0095 & 30 & 540217 & $1.851 \times 10^{-10}$ & $<1.0001$ \\
\hline
\end{tabular}

Table 11. Elliptic DE Mesh Parameters

\begin{tabular}{crlr|crcrr}
\hline Grid number & $N$ & $\Delta x_{N}$ & \multicolumn{1}{l}{$\Delta x_{0}$} & Grid number & $N$ & $\Delta x_{N}$ & $\Delta x_{0}$ \\
\hline 1 & 5 & $1.6667 \mathrm{E}-01$ & $1.7510 \mathrm{E}-05$ & 16 & 1851 & $5.3996 \mathrm{E}-04$ & $3.6474 \mathrm{E}-07$ \\
2 & 7 & $1.2500 \mathrm{E}-01$ & $4.9805 \mathrm{E}-05$ & 17 & 2776 & $3.6010 \mathrm{E}-04$ & $2.4303 \mathrm{E}-07$ \\
3 & 10 & $9.0909 \mathrm{E}-02$ & $5.5979 \mathrm{E}-05$ & 18 & 4164 & $2.4010 \mathrm{E}-04$ & $1.6195 \mathrm{E}-07$ \\
4 & 15 & $6.2500 \mathrm{E}-02$ & $4.5053 \mathrm{E}-05$ & 19 & 6246 & $1.6008 \mathrm{E}-04$ & $1.0793 \mathrm{E}-07$ \\
5 & 22 & $4.3478 \mathrm{E}-02$ & $3.2401 \mathrm{E}-05$ & 20 & 9369 & $1.0672 \mathrm{E}-04$ & $7.1938 \mathrm{E}-08$ \\
6 & 33 & $2.9412 \mathrm{E}-02$ & $2.1768 \mathrm{E}-05$ & 21 & 14053 & $7.1154 \mathrm{E}-05$ & $4.7953 \mathrm{E}-08$ \\
7 & 49 & $2.0000 \mathrm{E}-02$ & $1.4530 \mathrm{E}-05$ & 22 & 21079 & $4.7438 \mathrm{E}-05$ & $3.1967 \mathrm{E}-08$ \\
8 & 73 & $1.3514 \mathrm{E}-02$ & $9.6318 \mathrm{E}-06$ & 23 & 31618 & $3.1627 \mathrm{E}-05$ & $2.1310 \mathrm{E}-08$ \\
9 & 109 & $9.0909 \mathrm{E}-03$ & $6.3768 \mathrm{E}-06$ & 24 & 47427 & $2.1085 \mathrm{E}-05$ & $1.4206 \mathrm{E}-08$ \\
10 & 163 & $6.0976 \mathrm{E}-03$ & $4.2254 \mathrm{E}-06$ & 25 & 71140 & $1.4057 \mathrm{E}-05$ & $9.4705 \mathrm{E}-09$ \\
11 & 244 & $4.0816 \mathrm{E}-03$ & $2.8036 \mathrm{E}-06$ & 26 & 106710 & $9.3711 \mathrm{E}-06$ & $6.3136 \mathrm{E}-09$ \\
12 & 366 & $2.7248 \mathrm{E}-03$ & $1.8600 \mathrm{E}-06$ & 27 & 160065 & $6.2474 \mathrm{E}-06$ & $4.2090 \mathrm{E}-09$ \\
13 & 549 & $1.8182 \mathrm{E}-03$ & $1.2358 \mathrm{E}-06$ & 28 & 240097 & $4.1650 \mathrm{E}-06$ & $2.8060 \mathrm{E}-09$ \\
14 & 823 & $1.2136 \mathrm{E}-03$ & $8.2249 \mathrm{E}-07$ & 29 & 360145 & $2.7767 \mathrm{E}-06$ & $1.8706 \mathrm{E}-09$ \\
15 & 1234 & $8.0972 \mathrm{E}-04$ & $5.4769 \mathrm{E}-07$ & 30 & 540217 & $1.8511 \mathrm{E}-06$ & $1.2471 \mathrm{E}-09$ \\
\hline
\end{tabular}




\section{References}

${ }^{1}$ Knupp, P. M. and Salari, K., Verification of Computer Codes in Computational Science and Engineering, Chapman \& Hall/CRC, 2003.

${ }^{2}$ Roache, P. J., Verification and Validation in Computational Science and Engineering, Hermosa Publishers, 1998.

${ }^{3}$ Salari, K. and Knupp, P., "Validation by the Method of Manufactured Solutions," Tech. Rep. SAND2000-1444, Sandia National Labs., June 2000.

${ }^{4}$ Oberkampf, W. and Trucano, T., "Verification and Validation in Computational Fluid Dynamics," Tech. Rep. SAND2002059, Sandia National Labs., 2002.

${ }^{5}$ Roy, C., McWherter-Payne, M., and Oberkampf, W., "Verification and Validation for Laminar Hypersonic Flowfields Part 1: Verification," AIAA Journal, Vol. 41, No. 10, 2003, pp. 1934-1943.

${ }^{6}$ Hoffman, J., "Relationship between the Truncation Errors of Centered Finite-Difference Approximations on Uniform and Nonuniform Meshes," J. Comp. Phys., Vol. 46, 1982, pp. 469-474.

${ }^{7}$ Roe, P. L., "Approximate Riemann Solvers, Parameter Vectors, and Difference Schemes," J. Comp. Phys., Vol. 43, 1981, pp. 357-372.

${ }^{8}$ van Leer, B., "Towards the Ultimate Conservative Difference Schemes V. A second Order Sequel to Godunov's Method," J. Comp. Phys., Vol. 32, 1979, pp. 101-136. 